\title{
The equivariant universality and couniversality of the Cantor cube
}

\author{
by \\ Michael G. Megrelishvili (Ramat-Gan) and Tzvi Scarr (Jerusalem)
}

\begin{abstract}
Let $\langle G, X, \alpha\rangle$ be a $G$-space, where $G$ is a non-Archimedean (having a local base at the identity consisting of open subgroups) and second countable topological group, and $X$ is a zero-dimensional compact metrizable space. Let $\left\langle H\left(\{0,1\}^{\aleph_{0}}\right),\{0,1\}^{\aleph_{0}}, \tau\right\rangle$ be the natural (evaluation) action of the full group of autohomeomorphisms of the Cantor cube. Then

(1) there exists a topological group embedding $\varphi: G \hookrightarrow H\left(\{0,1\}^{\aleph_{0}}\right)$;

(2) there exists an embedding $\psi: X \hookrightarrow\{0,1\}^{\aleph_{0}}$, equivariant with respect to $\varphi$, such that $\psi(X)$ is an equivariant retract of $\{0,1\}^{\aleph_{0}}$ with respect to $\varphi$ and $\psi$.
\end{abstract}

1. Introduction. The Cantor cube $\mathcal{C}=\{0,1\}^{\aleph_{0}}$ is a universal space in the class of zero-dimensional, separable, metrizable spaces, that is, every such space can be topologically embedded into $\mathcal{C}$. In particular, every compact, zero-dimensional, metrizable space is homeomorphic to a closed subset of $\mathcal{C}$. Sierpiński [15] showed that every non-empty closed subset of $\mathcal{C}$ is a retract of $\mathcal{C}$. This gives us the following well-known fact.

FACT 1.1. Every non-empty, compact, zero-dimensional, metrizable space is homeomorphic to a retract of $\mathcal{C}$.

Our Main Theorem 3.5 is an equivariant generalization of Fact 1.1 for non-Archimedean acting groups. A topological group is non-Archimedean if it has a local base at the identity consisting of open subgroups. The class of non-Archimedean groups includes:

2000 Mathematics Subject Classification: 54H15, 22 A99.

Key words and phrases: Cantor cube, $G$-compactification, universal space, non-Archimedean group.

Fragments of this work are part of the second author's Ph.D. dissertation at Bar-Ilan University, under the supervision of Professor Hillel Furstenberg. 
- the prodiscrete (in particular, the profinite) groups;

- the groups arising in non-Archimedean functional analysis [14] (for example, the additive groups of the fields of $p$-adic numbers);

- the group $\operatorname{Is}(X, d)$ of all isometries of an ultrametric space $(X, d)$, with the topology of pointwise convergence;

- the locally compact, totally disconnected groups [3];

- the symmetric group $S_{\infty}$ on a countably infinite set, with the topology of pointwise convergence;

- the full group $H(X)$ of autohomeomorphisms of $X$, with the compactopen topology, where $X$ is a compact Hausdorff zero-dimensional space (see Lemma 3.2 below).

In fact, a topological group $G$ is non-Archimedean iff $G$ is a topological subgroup of $H(X)$ for some appropriate compact Hausdorff zero-dimensional space $X$. This complete characterization of the non-Archimedean groups is a part of Theorem 3.3 below. It is easy to show that the class of all nonArchimedean groups is a variety in the sense of [12]. That is, this class is closed under the formation of topological subgroups, products and quotient groups.

Note that the transformation groups having zero-dimensional (in particular, ultrametric) phase spaces have many applications in descriptive set theory $[1,6,7]$.

2. Preliminaries and conventions. All topological spaces in this paper are assumed to be Hausdorff. The neutral element of a group $G$ is denoted by $e_{G}$. The weight $w(X)$ of a topological space $X$ is defined to be $\tau(X) \cdot \aleph_{0}$, where $\tau(X)$ denotes the minimal cardinality of a base for $X$.

For information on uniform spaces, we refer the reader to [4]. If $\mu$ is a uniformity for $X$, then the collection of elements of $\mu$ which are finite coverings of $X$ forms a base for a uniformity for $X$, which we denote by $\mu_{\text {fin }}$. If $(X, \mu)$ is a uniform space, the uniform completion $\left(\widehat{X}, \widehat{\mu}_{\text {fin }}\right)$ of $\left(X, \mu_{\text {fin }}\right)$ is a compact uniform space known as the Samuel compactification of $(X, \mu)$. A partition of a set $X$ is a covering of $X$ consisting of pairwise disjoint subsets of $X$. Following [14], we say that a uniform space $(X, \mu)$ is non-Archimedean if it has a base consisting of partitions of $X$. Equivalently, $\mu$ is generated by a system $\left\{d_{i}\right\}$ of ultrapseudometrics, that is, pseudometrics, each of which satisfies the strong triangle inequality $d_{i}(x, z) \leq \max \left\{d_{i}(x, y), d_{i}(y, z)\right\}$. Clearly, a non-Archimedean uniform space is zero-dimensional in the uniform topology. A topological group is non-Archimedean iff its right uniformity is nonArchimedean.

The following result is well known (see, for example, [4] and [5]). 
Lemma 2.1. Let $(X, \mu)$ be a non-Archimedean uniform space. Then both $\left(X, \mu_{\mathrm{fin}}\right)$ and the uniform completion $(\widehat{X}, \widehat{\mu})$ of $(X, \mu)$ are non-Archimedean uniform spaces.

A topological transformation group, or $G$-space, is a triple $\langle G, X, \alpha\rangle$, where $G$ is a topological group (called the acting group), $X$ is a topological space (called the phase space), and $\alpha: G \times X \rightarrow X$ is a continuous action. For each $g \in G$, the $g$-transition map is the function $\alpha^{g}: X \rightarrow X$, $\alpha^{g}(x)=g x$.

Definition 2.2. Let $\left\langle G_{1}, X_{1}, \alpha_{1}\right\rangle$ be a $G_{1}$-space, and let $\left\langle G_{2}, X_{2}, \alpha_{2}\right\rangle$ be a $G_{2}$-space. Suppose that $\varphi: G_{1} \hookrightarrow G_{2}$ is a topological group embedding.

(1) A continuous function $\psi: X_{1} \rightarrow X_{2}$ is equivariant with respect to $\varphi$ (or, simply, equivariant, if $\varphi$ is clear from the context) if, for all $g \in G_{1}$ and $x \in X_{1}, \psi(g x)=\varphi(g) \psi(x)$.

(2) Let $\psi: X_{1} \rightarrow X_{2}$ be an equivariant embedding with respect to $\varphi$. We say that $\psi\left(X_{1}\right)$ is an equivariant retract of $X_{2}$ (with respect to $\varphi$ and $\psi)$ if there is a continuous retraction $r: X_{2} \rightarrow \psi\left(X_{1}\right)$ which is equivariant with respect to $\varphi^{-1}: \varphi\left(G_{1}\right) \rightarrow G_{1}$.

Let $\langle G, X, \alpha\rangle$ be a $G$-space. If $\langle G, Y, \gamma\rangle$ is a compact Hausdorff $G$-space and $\psi: X \rightarrow Y$ is equivariant, then $Y$ is called a $G$-compactification of $X$. If, in addition, $\psi$ is a topological embedding, then $Y$ is a proper $G$-compactification of $X$. A $G$-space $\langle G, X, \alpha\rangle$ is $G$-Tikhonov if it has a proper $G$-compactification. Not every Tikhonov $G$-space is $G$-Tikhonov [8]. De Vries [19] proved that if $G$ is locally compact, then every Tikhonov $G$ space is $G$-Tikhonov. For every $G$-space $X$ there exists a (possibly improper) maximal $G$-compactification $\beta_{G} X$ (see [18]). For more information on $G$ compactifications, as well as for a general method of constructing Tikhonov $G$-spaces which are not $G$-Tikhonov, see [11].

Let $G$ be a topological group. Recall [2] that the collection of coverings $\{U x: x \in G\}$, where $U$ is a neighborhood of $e_{G}$, forms a base for the right uniformity $\mu_{\mathrm{R}}$ for $G$. In 1957, Teleman [16] proved that for arbitrary Hausdorff $G$, the Samuel compactification $\widehat{G}$ of $G$ with respect to its right uniformity is a proper $G$-compactification of the $G$-space $\left\langle G, G, \alpha_{\mathrm{L}}\right\rangle$, where $\alpha_{\mathrm{L}}$ is the usual left action of $G$ on itself. In fact, $\widehat{G}$ is isomorphic to $\beta_{G} G$ and is called the greatest ambit (see, for example, $[20]$ ). $\beta_{G} G$ is the maximal proper $G$-compactification of $\left\langle G, G, \alpha_{\mathrm{L}}\right\rangle$.

To the best of our knowledge, very little is known about the dimension of $\beta_{G} X$. Some special results can be found in $[8,9]$. The dimension of the greatest ambit $\beta_{G} G$ may be greater than the topological dimension of $G$ (simply take a cyclic dense subgroup $G$ of the circle group; then $\operatorname{dim} G=0$ and $\left.\operatorname{dim} \beta_{G} G=1\right)$. However, in the case of the Euclidean group $G=\mathbb{R}^{n}$, 
we have $\operatorname{dim} \beta_{G} G=\operatorname{dim} G$. This follows from Theorem 5.12 of [4]. By a result of Pestov [13], one has $\operatorname{dim} \beta_{G} G=0$ iff $G$ is non-Archimedean. An alternative proof of this will be given in Theorem 3.3 below.

\section{Proof of the main results}

FACT 3.1 ([9]). Every $G$-Tikhonov $G$-space $X$ has a proper $G$-compactification $Y$ such that $w(Y) \leq w(X) \cdot w(G)$ and $\operatorname{dim} Y \leq \operatorname{dim} \beta_{G} X$.

LEMma 3.2. If $X$ is a compact Hausdorff zero-dimensional space, then $H(X)$ is a non-Archimedean group.

Proof. For each two-element compact clopen partition $\left\{K_{1}, K_{2}\right\}$ of $X$, define

$$
B\left(K_{1}, K_{2}\right)=\left\{\varphi \in H(X): \varphi\left(K_{1}\right)=K_{1}, \varphi\left(K_{2}\right)=K_{2}\right\} .
$$

Let $\mathcal{B}=\left\{B\left(K_{1}, K_{2}\right):\left\{K_{1}, K_{2}\right\}\right.$ is a compact clopen partition of $\left.X\right\}$. Then $\mathcal{B}$ is a local base at $e_{H(X)}$ consisting of clopen subgroups, and, hence, $H(X)$ is non-Archimedean.

The following theorem provides a useful characterization of non-Archimedean groups. (As noted before, the equivalence of (i) and (ii) was established by Pestov [13].)

THEOREM 3.3. The following assertions are equivalent:

(i) $G$ is a non-Archimedean topological group;

(ii) $\operatorname{dim} \beta_{G} G=0$;

(iii) $G$ is a topological subgroup of $H(X)$ for some compact Hausdorff zero-dimensional space $X$ such that $w(X)=w(G)$.

Proof. (i) $\Rightarrow$ (ii). Suppose $G$ is non-Archimedean. Then the right uniformity $\mu_{\mathrm{R}}$ for $G$ is a non-Archimedean uniformity. By Lemma 2.1, the precompact uniformity $\left(\mu_{\mathrm{R}}\right)_{\text {fin }}$ for $G$ is also a non-Archimedean uniformity. Let $(\widehat{G}, \widehat{\mu})$ be the uniform completion of $\left(G,\left(\mu_{\mathrm{R}}\right)_{\text {fin }}\right)$. Then, again by Lemma $2.1, \widehat{\mu}$ is a non-Archimedean uniformity, and, hence, $\widehat{G}$ is zero-dimensional. But $(\widehat{G}, \widehat{\mu})$ is exactly $\beta_{G} G$.

(ii) $\Rightarrow$ (iii). By Fact 3.1 , there exists a zero-dimensional proper $G$-compactification $\left\langle G, X, \alpha_{\mathrm{L}}^{*}\right\rangle$ of $\left\langle G, G, \alpha_{\mathrm{L}}\right\rangle$ such that $w(X)=w(G)$. Let $\psi: G \rightarrow X$ be the corresponding equivariant embedding.

We will show that the map $\varphi: G \rightarrow H(X)$ defined by $\varphi(g)=\left(\alpha_{\mathrm{L}}^{*}\right)^{g}$ is a topological group embedding. Observe that $\varphi$ is one-to-one because $\alpha_{\mathrm{L}}^{*}$ extends the action $\alpha_{\mathrm{L}}$. To prove the continuity of $\varphi$, suppose $\alpha^{g} \in O=\{f \in$ $H(X): f(K) \subseteq U\}$, where $K \subseteq X$ is compact and $U \subseteq X$ is open. Using the compactness of $K$ and the continuity of $\alpha_{\mathrm{L}}^{*}$, we can find a neighborhood $V$ of $g$ such that $\varphi(V) \subseteq O$. Hence, $\varphi$ is continuous. 
It remains to show that if $O \subseteq G$ is open, then $\varphi(O)$ is open in $\varphi(G)$. Let $O \subseteq G$ be open. Then $\psi(O)$ is open in $\psi(G)$. Let $W \subseteq X$ be open such that $\psi(O)=W \cap \psi(G)$. Define $B=\left\{f \in H(X): f\left(\psi\left(e_{G}\right)\right) \in W\right\}$. Then $B$ is open in $H(X)$ and $\varphi(O)=B \cap \varphi(G)$. Hence, $\varphi(O)$ is open in $\varphi(G)$.

(iii) $\Rightarrow$ (i) follows directly by Lemma 3.2 .

FACT 3.4 (Brouwer). The Cantor cube $\{0,1\}^{\aleph_{0}}$ is the unique (up to homeomorphism) non-empty, compact, metrizable, zero-dimensional, perfect space.

Now we are ready to prove our main result.

TheOREm 3.5. Let $G$ be a non-Archimedean and second countable group, and let $X$ be a compact, metrizable, zero-dimensional $G$-space. Then

(1) there exists a topological group embedding $\varphi: G \hookrightarrow H(\mathcal{C})$;

(2) there exists an embedding $\psi: X \hookrightarrow \mathcal{C}$, equivariant with respect to $\varphi$, such that $\psi(X)$ is an equivariant retract of $\mathcal{C}$ with respect to $\varphi$ and $\psi$.

Proof. By Theorem 3.3, there exists a compact, second countable (and thus metrizable) zero-dimensional space $Y$ such that $H(Y)$ contains $G$ as a topological subgroup. We may as well assume that all homeomorphisms of $Y$ corresponding to elements of $G$ transform a certain base point $y_{0} \in Y$ onto itself (if not, replace $Y$ with a disjoint union $Y \cup\left\{y_{0}\right\}$ and redefine those homeomorphisms in an obvious way).

Let us identify the action of $G$ on $X$ with a homomorphism $w: G \rightarrow$ $H(X)$, and let $\mathcal{D}$ be a copy of the Cantor set. By Brouwer's theorem, the space $\mathcal{C}=X \times Y \times \mathcal{D}$ is homeomorphic to the Cantor set, and, clearly, the $\operatorname{map} \varphi: G \rightarrow H(\mathcal{C})$,

$$
g \mapsto\left(w(g), g, \operatorname{id}_{D}\right) \in H(X) \times H(Y) \times H(\mathcal{D}) \subseteq H(\mathcal{C}),
$$

is a continuous homomorphism, thus turning $\mathcal{C}$ into a $G$-space. This homomorphism is also an embedding, for its composition with the projection onto $H(Y)$ is the identity mapping, so it is one-to-one and the inverse is continuous.

We define $\psi: X \rightarrow \mathcal{C}$ by $x \mapsto\left(x, y_{0}, d_{0}\right)$, where $d_{0} \in \mathcal{D}$ is any base point, and the retraction $r: \mathcal{C} \rightarrow \psi(X)$ by $r(x, y, d)=\left(x, y_{0}, d_{0}\right)$. Then $\psi$ and $r$ are equivariant, and the proof is complete.

Theorem 3.6. $H(\mathcal{C})$ is universal in the class of all non-Archimedean, second countable groups, that is, every such group is topologically isomorphic to a subgroup of $H(\mathcal{C})$.

Final Remarks. (1) By Theorem 1.5.1 of [1], the group $S_{\infty}$ is also universal in this class.

(2) The group $H\left(I^{\aleph_{0}}\right)$ is universal in the class of all second countable topological groups, where $I$ is the closed interval $[0,1]$ (see [17]). Moreover, 
by [10], the topological transformation group $\left\langle H\left(I^{\aleph_{0}}\right), I^{\aleph_{0}}\right\rangle$ is universal in the class of all compact, metrizable $G$-spaces with second countable acting group $G$.

(3) The action on $\mathcal{C}$ which we defined in the proof of Theorem 3.5 intrinsically depends on the original action of $G$ on $X$, as the following example shows.

ExAmple 3.7. Let $\alpha: S_{\infty} \times \mathcal{C} \rightarrow \mathcal{C}$ be the natural "permutation of coordinates" action

$$
\alpha\left(g,\left(x_{n}\right)\right)=\left(x_{g(n)}\right) .
$$

Let $\overline{0}$ and $\overline{1}$ denote the two constant sequences of $\mathcal{C}$. Let $H=\{\overline{0}, \overline{1}\} \subseteq \mathcal{C}$. Consider $H$ as an $S_{\infty}$-subspace of $\mathcal{C}$.

Claim. $H$ is not an equivariant retract of $\mathcal{C}$ with respect to $\varphi=\operatorname{id}_{S_{\infty}}$ and $\psi=\mathrm{id}_{H}$.

Proof. The Cantor cube $\mathcal{C}$ is an $S_{\infty}$-ambit under the action $\alpha$, that is, it contains a point whose orbit is dense in $\mathcal{C}$. In fact, all points which contain infinitely many 0 's and infinitely many 1 's have dense orbits. Hence, every image of $\mathcal{C}$ under an equivariant map is also an $S_{\infty}$-ambit. However, $H$ is not an $S_{\infty}$-ambit.

Acknowledgments. The authors would like to thank the referees for many constructive suggestions and for a very substantial shortening of the proof of the Main Theorem.

\section{References}

[1] H. Becker and A. Kechris, The Descriptive Set Theory of Polish Group Actions, Cambridge Univ. Press, Cambridge, 1996.

[2] R. Engelking, General Topology, PWN, Warszawa, 1977.

[3] E. Hewitt and K. A. Ross, Abstract Harmonic Analysis, Vol. 1, Springer, 1963.

[4] J. Isbell, Uniform Spaces, Amer. Math. Soc., Providence, 1964.

[5] - Zero-dimensional spaces, Tôhoku Math. J. (2) 7 (1955), 1-8.

[6] A. Kechris, Classical Descriptive Set Theory, Springer, New York, 1995.

[7] -, Descriptive dynamics, in: Descriptive Set Theory and Dynamical Systems, London Math. Soc. Lecture Note Ser. 277, Cambridge Univ. Press, 2000, 231-258.

[8] M. Megrelishvili, A Tikhonov G-space that does not have compact G-extension and G-linearization, Russian Math. Surveys 43 (1988), 177-178.

[9] - Compactification and factorization in the category of $G$-spaces, in: Categorical Topology and its Relation to Analysis, Algebra and Combinatorics, J. Adámek and S. MacLane (eds.), World Sci., Singapore, 1989, 220-237.

[10] —, Free topological G-groups, New Zealand J. Math. 25 (1996), 59-72.

[11] M. Megrelishvili and T. Scarr, Constructing Tychonoff $G$-spaces which are not $G$ Tychonoff, Topology Appl. 86 (1998), 69-81. 
[12] S. A. Morris, Varieties of topological groups (a survey), Colloq. Math. 46 (1982), 147-165.

[13] V. G. Pestov, On free actions, minimal flows, and a problem by Ellis, Trans. Amer. Math. Soc. 350 (1998), 4149-4165.

[14] A. C. M. van Rooij, Non-Archimedean Functional Analysis, Monogr. Textbooks Pure Appl. Math., Marcel Dekker, 1978.

[15] W. Sierpiński, Sur les projections des ensembles complémentaires aux ensembles (A), Fund. Math. 11 (1928), 117-122.

[16] S. Teleman, Sur la représentation linéaire des groupes topologiques, Ann. Sci. École Norm. Sup. 74 (1957), 319-339.

[17] V. V. Uspenskiǔ, A universal topological group with a countable basis, Funct. Anal. Appl. 20 (1986), 160-161.

[18] J. de Vries, Topological Transformation Groups I, Mathematical Centre Tracts 65, Mathematisch Centrum, Amsterdam, 1975.

[19] —, On the existence of G-compactifications, Bull. Acad. Polon. Sci. Sér. Sci. Math. Astronom. Phys. 26 (1978), 275-280.

[20] - Elements of Topological Dynamics, Kluwer, Dordrecht, 1993.

Department of Mathematics

Bar-Ilan University

52900 Ramat-Gan, Israel

E-mail: megereli@macs.biu.ac.il

Web: http://www.cs.biu.ac.il/ ${ }^{\sim}$ megereli
Department of Applied Mathematics Jerusalem College of Technology 21 Havaad Haleumi St. Jerusalem, Israel 91160 E-mail: scarr@avoda.jct.ac.il

Received 2 March 2000;

in revised form 21 November 2000 